\title{
Electrification of urban mobility: the case of catenary-free buses
}

Riccardo Scarinci ${ }^{1}$ riccardo.scarinci@epfl.ch

Alessandro Zanarini ${ }^{2}$ alessandro.zanarini@ch.abb.com

Michel Bierlaire ${ }^{1} \quad$ michel.bierlaire@epfl.ch

Report TRANSP-OR 181030

30 October 2018

${ }_{1}$ École Polytechnique Fédérale de Lausanne (EPFL), School of Architecture, Civil and Environmental Engineering (ENAC), Transport and Mobility Laboratory (TRANSPOR), Switzerland, transp-or.epfl.ch

2 ABB Corporate Research Center, Baden, Switzerland 


\begin{abstract}
Electric mobility is now a reality, and services based on electric vehicles, such as electric car-sharing and electric buses, are becoming part of urban mobility. These new modes bring new challenges for the design and operations of the systems that require specific decision support tools for the evaluation of the service performance. In order to pave the way toward integrated electrified urban mobility, we need operational tools that enable the technology to be successfully implemented in practice. In this paper, we propose a simulation framework for the evaluation of electric vehicle operations. The framework is composed of a simplified traffic simulator, an energy consumption model, and a speed profile model. We estimate a macroscopic representation of the energy consumption derived from a nanosomic energy model. This allows a trade-off between accuracy and computation time. Then, we define a stochastic representation of the stopping profile, which is essential to derive the speed profile in case of missing data. The framework generates a distribution of the key performance indicators, like the battery state of charge, for a large number of scenarios. The utility of the framework is illustrated on the case study of a public transport service using catenary-free electric buses in combination with fast-charging stations. The results quantify the probability that the on-board batteries reach a low state of charge, and they evaluate the effects of different strategies to reduce this probability. This information is useful for transport planners to dimension the system correctly.
\end{abstract}

\title{
1 Introduction
}

The use of electric vehicles is increasing, especially in cities, thanks to recent developments in battery technology, political decisions and environmental awareness (Palomares et al., 2012). Private plug-in hybrid and electric cars are expected to cover up to $50 \%$ of the total vehicle market share (Fernandez-Antolin et al., 2016). Electric car-sharing systems are part of the urban mobility, and autonomous electric buses and dial-a-ride services will become a reality soon. However, there is still a long way to go until a comprehensive and integrated electric urban mobility system is fully in place.

In particular, these new services bring new challenges for the design and operations of electric transport systems. Elements, such as the dimension of the on-board batteries, the position of the charging stations and the location of parking places, should be designed carefully. Indeed, electric vehicles have long charging times or short ranges, and disruptions to the system could have catastrophic consequences on their operations. For example, due to unforeseen congestion, an electric bus could be unable to reach the next charging station. This leads to further delay for the passengers and costly recovery strategies to place the bus back to operation. It is clear that tools designed for the evaluation of services based on electric vehicles are needed. However, due to the novelty of these services, such tools have not been yet fully developed.

In this paper, we propose a simple and operational framework useful for the 
evaluation of electric vehicle operations. The framework is composed of the following elements: a simplified traffic simulator, an energy consumption model, and a speed profile model.

We simulate the electric vehicle operations, such as movements on the road network, stops at intersections and recharging at feeding stations. The framework focuses on the operations of the vehicles, and it does not consider the behavioral aspects of the demand such as route choice, destination choice and departure times. The traffic simulation allows for the evaluation of key performance indicators, such as the battery state of charge, in multiple scenarios including extreme cases. The results are essential to understand the robustness of the service, and they can be used to improve the planning of the system.

The framework can be applied to the evaluation of various versions or electric urban mobility, such as private vehicles, car-sharing and public transport services. However, the general framework presented in this article is inspired by a real project aimed at planning a service of catenary-free electric buses. The present paper illustrates the use of this framework on the operations of this type of electric mobility. These vehicles have a series of on-board batteries that combined with charging stations installed at specific bus stops allow the removal of the overhead electric cables, i.e. the catenary. The charging stations partially replenish the bus batteries in a few seconds at the bus stops.

The design of this simulation-based framework has several methodological challenges. (i) We need to identify which elements to model starting from the real operations of the vehicles, in this case a catenary-free bus service. (ii) For each element, we need to define the simulation scale, i.e. macro-, meso-, micro- or nanoscopic, and the dynamics to be considered. (iii) We need to structure a simulation that is robust to missing data. The simulation must be usable from the early stage of the planning (characterized by low information and missing data) until the final stage of the planning.

To address these challenges, we develop a simulation framework that includes macroscopic elements like traffic flow, microscopic elements like individual passengers, and elements represented by random variables like the probability of stopping at intersections. In addition, an interface with nanoscopic energy consumption models is implemented.

The paper is structured as follows. Section 2 reviews existing approaches used to simulate the main components of electric vehicles and their operations. We specify the requirement that transportation planners need from a simulation tool in Section 3. The structure of the simulation framework is presented in Section 4. We describe two specific aspects of the framework, namely energy consumption and representation of the stopping profile, in Section 5 and Section 6 , respectively. The simulation is illustrated on a concrete example of a catenary-free bus line in Section 7, and the main conclusions are reported in Section 8. 


\section{Literature review}

Design and optimization of electric vehicle operations are widely studied, both for private and public services (Silvas et al., 2017). For private mobility, the research focuses on the interaction between mobility patterns (demand) and availability of charging stations (supply). We refer to Rahman et al. (2016) for a recent review. He et al. (2013) investigate the optimal deployment of public charging stations considering not only demand and supply, but also including the price of electricity. They study this interaction using mathematical programming. Hess et al. (2012) propose a solution for optimal placement of charging stations using a combination of genetic programming and simulation of the vehicle dynamics. Frade et al. (2011) show that the optimal configuration of charging stations in a real case study strongly depends on the mobility patterns.

The planning of public transport services is also widely studied, and it encloses a variety of fields (Banister, 2001; Black, 2018). These fields range from transit network design (TND) at the macroscopic scale to nanoscopic models of the vehicle operations. TND aims at identifying the optimal configuration of routes, frequencies and timetables (Guihaire and Hao, 2008). A vast number of mathematical formulations have been proposed from mixed integer non-linear programming (Deb and Chakroborty, 1998; Jansen et al., 2002) to robust optimization framework (Nikolic and Teodorovic, 2013; Cadarso et al., 2017). These methods often represent aggregated demand and supply, they are used at the planning phase and ignore the operational phase. For a comprehensive review of this topic we refer to Desaulniers and Hickman (2007), Fan and Machemehl (2006) and Farahani et al. (2013).

In recent years, the topic of catenary-free vehicles has been increasingly studied. Kuhne (2010) reviews the technical developments in the energy saving induced by this technology. Cimini et al. (2017) propose a model predictive control (MPC) approach to suggest the optimal speed profile. Chen et al. (2014) and Lurkin et al. (2018) focus on catenary-free bus planning. They develop an optimization model considering the join decisions of vehicle characteristics, like the battery type, and infrastructural characteristics, like charging station locations.

With the increased interest in electric vehicles, numerous energy consumption models have been developed (Moreno et al., 2006; Ehsani et al., 2018). They can be classified into two types. Fully empirical models based on measurement data, and semi-empirical models based on physical representation of the system (Punpaisan and Kulworawanichpong, 2014; Gao et al., 2007). Several models are developed from real case studies, such as in Portugal (Perrotta et al., 2014), United States (Filippo et al., 2014) and Finland (Erkkila et al., 2013).

An increasing number of energy consumption models include aspects related to the entire life-cycle of the battery, such as aging and degradation (Dunn et al., 2015). For example, Hu et al. (2015) include a battery health model in the dimensioning decision of a bus energy storage system. Shirk and Wishart (2015) study the effect of fast charging on the battery life.

Recently, energy consumption models are integrated into traffic simulation models to investigate the consequence of the vehicle operations. For example, 
Yang et al. (2013) use a microscopic car-following model to evaluate the vehicle energy consumption due to traffic. Tang et al. (2015) propose a new car-following model considering the influences of the driving range on the driving behavior.

Traffic simulation uses a road network as an input to simulate the vehicle dynamics. However, at the early stage of the planning process, the road network and the driver routes are not fully defined. A possibility to cope with this lack of information is to use a meta-representation of the network. Several studies investigates the meta-characteristics of road networks in urban areas. Jacobs (1993), Cardillo et al. (2006), and Crucitti et al. (2006) compare systematically the dimensions, plans and patterns of roads in different cities. Peponis et al. (2007) measure block size, street density and intersection density in 12 large metropolitan regions. Marshall (2004) and Jiang and Claramunt (2004) study urban layouts considering both transport and urban planning issues. From these studies, we can identify the minimum and maximum average distance between two intersections. It appears that for European cities the average is between 31 and 112 meters, for Venice and Barcelona respectively. However, none of the studies describes the probability distribution of the intersections useful to simulate the speed profile at an early planning stage. We discuss this aspect in Section 6.

This review section shows the extent of the research fields included in the planning, simulation and optimization of services based on electric vehicles. In the rest of the paper, we introduce a simulation framework that clarifies the position of the different components and the possible integration among them.

\section{$3 \quad$ Functional requirements}

Transportation planners need flexible tools for supporting the dimensioning and operation decisions of existing and future services. Dimensioning decisions are aspects of the system that are fixed over a long period of time, such as infrastructure characteristics (e.g. charging station locations and on-board battery types). Operational decisions, for example the time schedule, can be changed more frequently, and they depend on the daily operations of the system, such as traffic state and passenger demand.

Operational decisions depends on factors with a high degree of uncertainty, and the combination of adverse events could lead to a malfunction of the system. For example, an electric bus following the usual bus schedule could run out of energy in a day with unexpected high temperature and a high passenger demand due to an extreme use of the air conditioning system. The probability of these extreme events should be considered in a decision support tool, and the risk associated with specific dimensioning decisions of the system should be quantified.

Traffic simulation is widely used to study the performance of complex transportation systems under different scenarios and to evaluate consequences of extreme events (Barcelo, 2010). The main advantage of using simulation is that the distribution of key performance indicators (KPI) can be evaluated, and not only a single value. This is done by describing the inputs, i.e. the operational characteristics in our case, with their realization probability. Then, multiple runs of 
the simulation are performed, and the distribution of KPIs evaluated (Bierlaire, 2015).

We identify three main requirements that should be included in a simulation for planning transport services based on electric vehicles. The simulation should be:

- usable in different planning stages,

- integrated with other components,

- capable of evaluating a vast range of scenarios.

We use the example of a catenary-free bus service to clarify these concepts. However, the same concepts can be extended to other electric vehicle services such as electric car-sharing systems or autonomous electric dial-a-ride services.

Different planning stages. We can divide the planning process into three stages: early, medium and final. The early stage is characterized by a high uncertainty on the transport demand, type of vehicles used by the system and, specifically for public transport, the location of bus lines and their routes. For example, the planners have an estimate of the route followed by the bus lines, and the possible location of the bus stops in the early stage. However, essential information on the infrastructure, such as traffic conditions, intersection locations with bus priority and public transport dedicated lanes, are not known. The same uncertainty is present for the passenger demand. In the medium stage of planning, more precise information on supply and demand is available. For example, the bus lines are known as well as the expected flow of passengers between bus stops. In the final stage, all infrastructure and demand characteristics are well defined, or at least the maximum and minimum expected value of each aspect.

The traffic simulation used to support the planning of the system should provide different types of results in each stage. In the early stage, it should provide preliminary analysis to confirm the feasibility of the service. In the medium stage, the simulation should provide confidence intervals on the KPIs, and, in the final stage, it should be able to provide precise distributions of the KPI. These distributions allow planners to estimate the service level and costs, as well as evaluate the consequence of the dimensioning decisions.

Integration. As mentioned in Section 2, energy consumption models and optimization algorithms for dimensioning decisions are key components of the planning process, and they should be integrated into the traffic simulation.

Energy consumption models are particularly relevant in the final planning stage. Once a well-specified stretch of road has been identified by the transport planner as a potential bus route, the traffic simulation should be able to use an energy consumption model providing a precise battery consumption. To do so, the traffic simulation should generate an accurate speed profile to be evaluated by the energy consumption model. However, a single accurate speed profile does not exist due to the uncertainty of the operational characteristics such as passenger demand 
or traffic condition. To deal with this problem, the traffic simulation outputs different speed profiles for the possible scenarios, which are based on the stochastic variables representing the system. Then, a speed profile should be sampled from the generated profiles based on the information required by the operator. For example, to have a detailed information on the worst case, the slowest speed profile or the speed profile with the maximum number of stops should be sampled.

Note that high fidelity energy consumption models are often microscopic or nanoscopic. This implies long computation time that limits the possibility of evaluating a large number of different speed profiles. To overcome this limitation, a macroscopic representation of the bus energy consumption may be integrated in the traffic simulation. We describe such a procedure in Section 5.

The second tool that should be linked to the traffic simulation is the algorithm used for defining the dimensioning decisions. This component is more relevant to the early stage of planning, when several dimensioning decisions can be taken using optimization methods. For example the location of the feeding stations, the battery size and the type of chemistry used in the battery. Traditionally, optimization tools optimize the system on an average case or the worst scenario. However, this does not consider the uncertainty of the operational characteristics. The traffic simulation, evaluating multiple runs, can provide the empirical probability distribution of the KPIs. These distributions can be used by the optimization tool for stochastic optimization. Alternatively, the lowest and highest bounds of the distribution can be used for robust optimization.

Evaluate a vast range of scenarios. The vehicle dynamics and operations are subject to a high degree of uncertainty. For example, the traffic conditions in a daily commuting route change from one day to another, resulting in a substantially different speed profile. This is particularly true in the early planning stage where basic infrastructural configurations are unknown. To deal with this aspect, the traffic simulation should be able to evaluate a vast range of scenarios. Therefore, the inputs should be represented by stochastic distributions representing the probability of occurrence for each factor.

The most important factors are traffic conditions, passenger flow, weather conditions, driving style, sharp turns, intersection locations and probability of stopping. These factors influence the speed profile, the stop time at stations, the total weight of the bus and the auxiliary consumption (heating/cooling).

The main challenge associated with the requirement of evaluating a vast range of scenarios is the computation time. The simulation framework has multiple uncertain inputs, which lead to the need of a high number of runs to investigate the KPI distributions. In order to maintain a limited computation time, it is necessary to identify the appropriate level of detail for the models used in the traffic simulation. The most appropriate level is a trade-off between accuracy and computation time. To meet this trade-off, we propose an hybrid traffic simulation framework that mix macro-, micro- and nano-scopic elements. The scale chosen for each element is described in the next section. 


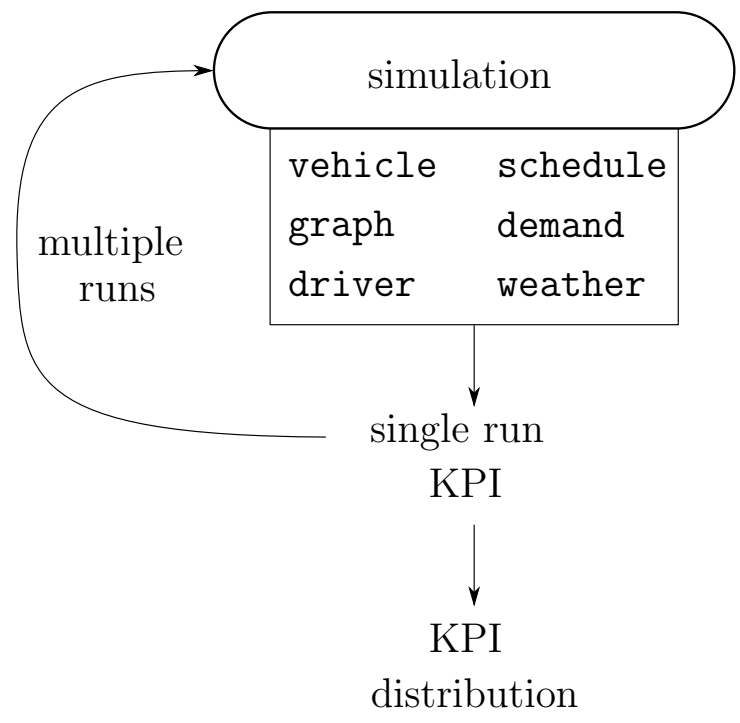

Figure 1: Main elements of the traffic simulation and multiple run structure.

\section{Simulation framework}

We chose to structure the simulation framework as a discrete-event simulation. This allows a flexible design that can be adapted for different modes of electric mobility, as well as having low computation time. In the following, we introduce the framework logic, the main elements, the multiple run simulation structure, the event logic, the input characteristics and the computation time performance. Once again, we use a catenary-free electric bus service as an example. Therefore, we introduce some elements specific of public transport services like stations, time schedule and passenger demand.

Main elements. The simulation framework is composed of several main elements interacting among them. Figure 1 provides a graphical representation of these elements. For clarity, we mark the main elements in a different font, e.g. vehicle, and we describe the logic of their interaction in details.

The main objective of the traffic simulation is to simulate the movement of vehicles in a road network. Each vehicle has associated characteristics, such as passenger capacity and maximum acceleration. These characteristics are often decision variables of the early and medium planning stages.

The road network and its elements are represented as a connected graph composed of nodes and arcs. Nodes represent bus stops and intersections, which are essential for calculating the speed profile (see Section 6). Given the specificity of a catenary-free bus service, each bus station can have multiple stands where vehicles embark and disembark passengers. Some stands are equipped with feeding stations, where vehicles charge the on-board batteries following predetermined charging policy. Arcs have associated attributes, such as the traffic conditions and speed limits.

Each vehicle has a driver with a specific driving style. Drivers have different characteristics, such as the desired speed and desired deceleration, which influence 
the speed profile and consequently the energy consumption.

Vehicles follow a public transport schedule. It contains the information on the public transport lines, bus stops and a timetable with the expected arrival and departure time from each stop. For the complex representation of the timetable, it is recommended to follow the General Transit Feed Specification (GTFS) standards (Antrim and Barbeau, 2013).

The movements of each vehicle are recorded as a series of changes in acceleration. These changes in acceleration are calculated using kinematic equations based on the stopping profile discussed in Section 6. This macroscopic representation of the movements is necessary to maintain a low complexity of the vehicle dynamics, which is needed for a low computation time.

While moving, the energy in the on-board battery of the vehicle is consumed or regenerated. The consumption is recorded as a series of changes in the power consumption. The energy consumption is also function of the weather conditions such as temperature, sun irradiation and cloud coverage. Given the importance of the energy consumption as a key performance indicators, the simulation uses two models, one macroscopic and one microscopic. We refer to Section 5 for details.

The demand of passengers is represented by an origin-destination with stops as origins and destinations. Passengers are represented as individual agents, with individual characteristic such as the travel path and the body weight (which is needed to estimate the energy consumption, see Section 5).

Multiple run simulation. The traffic simulation performs multiple runs of the same input varying the random seed for the random generator. Note that some components are simulation-specific, e.g. the vehicle capacity remain the same for all runs, and other components are run-specific, e.g. the number of passengers on-board may change from run to run. An element that is simulation specific is equal for all simulation runs. An element that is run-specific is different among runs, i.e. it is a realization from a random variable.

The results are used to compute the distribution of the KPIs, for example the distribution of minimal battery state of charge.

Event logic. The framework is a discrete event simulation, and the concept of "event" is at the core of the simulation. The state variables describing the status of the system, such as buttery charge or vehicle speed, are updated at the occurrence of specific events, and time is discrete.

The first events in the simulation are related to the generation of the vehicles at the depot and the arrival of the first passengers at the bus stops. These few initial events trigger the following events. For example, the arrival of a passenger, when executed by the simulation, triggers the arrival of the next passenger.

The main events associated with a bus are the arrival at the bus station indicated in its schedule, the identification of an available stand for embarking/disembarking passengers and recharging the on-board battery (if a feeding station is available), and the departure from the station. The difference between the schedule arrival and departure time are recorded to identify possible delay in the bus operations. 
Between two stations, the speed profile of the bus is calculated considering intersections and traffic conditions influencing the acceleration and speed of the vehicles. The battery consumption, or regeneration when breaking or downhills, is calculated by passing the speed profile to the nanoscopic battery model, or using the macroscopic battery model described in Section 5 .

The simulation of the bus movements continues until the schedule of all buses is completed. The KPIs of each run is then recorded, for example, the minimum state of charge for each vehicle during the entire operation of the bus, and the KPI distribution is estimated based on all runs.

Input and characteristics. To compensate for the lack of information, several input elements are described by their statistical properties. Empirical distributions from the literature are used for discrete and continuous random variables representing the elements of the system. For example, each passenger is generated with a weight drawn from a distribution representing the general population weight (Walpole et al., 2012). Then, the total weight of the passengers on board, a crucial factor influencing the energy consumption, is calculated as the sum of the individual passenger weights. The same approach is used for describing the drivers' driving style, which is characterized by the desired acceleration and maximum speed.

Another stochastic input is the demand, for example, the arrival time of passengers at the bus stop, or the request time and location for a car-sharing system. We use homogeneous and non-homogeneous stochastic Poisson processes to mimic passenger arrivals and embarking (Ross, 2013).

Computation time. The framework of the simulation is structured to allow multiple threads. Each simulation run can be run independently from the others. The generation of the run-specific random seeds from the simulation random seed is the only link among runs. Every time that the execution of a run is completed, the results are stored in the simulation together with the results of the other runs. This structure reduces the simulation time required to evaluate multiple runs using parallel computing.

In the following two sections, we focus on two aspects of this general framework that received less attention from the scientific community. Specifically, we describe a possible macroscopic representation of the vehicle energy consumption suitable for integration in the traffic simulation, and a stochastic representation of the stopping profile caused by intersections useful at the early planning stage.

\section{$5 \quad$ Energy consumption model}

Accurate energy consumption models often have a detailed representation of the physical components of the vehicle that influence the energy consumption. Examples of these components are the traction system, battery size (as the number of cells in series and strings in parallel), the cell chemistry, and the type of auxiliaries for cooling and heating. Given the high details of the represented elements, these 
models can be classified as nanoscopic (Perrotta et al., 2014). They are used to give a precise indication of the energy consumption of the vehicle for a specific set of dimensioning decisions and operational characteristics.

This type of nanoscopic energy consumption models cannot be used directly in the traffic simulation due to the computationally expensive performance. To decrease the computation time, traffic simulation tools usually incorporate macroscopic energy consumption models from the literature (Larminie and Lowry, 2004). The main drawback of this approach is that these energy consumption models are general. They do not represent the vehicle chosen as a dimensioning decision by the transport planners, which need to evaluate the performance of specific vehicles on a specific services. Producers of electric vehicles have empirical data and accurate nanoscopic models providing precise information specifically for the chosen vehicles. These data and models should be used to generate a macroscopic model of the energy consumption of specific vehicles. Then, this macroscopic model should be used in the traffic simulation.

To test this approach, we estimate a macroscopic energy consumption model from a nanoscopic model of the vehicle used in our case study. In the following, we introduce the chosen model, and we report the results of the estimation.

Nanoscopic energy consumption model. The nanoscopic energy consumption model used is a deterministic model of a catenary-free electric bus developed by Horsky (2018), which has 133 input parameters. With respect to the traffic simulation, some of these parameters are fixed, i.e. they do not change during daily operations, for examples the traction system, auxiliary type and battery specifications. Others vary dynamically during the traffic simulation, such as the number of passengers, street slope and speed. For each parameters that may change during a traffic simulation, we identify a lower and an upper bound, as well as an evaluation step. We run several scenarios varying the values of these parameters, and we record the energy consumption returned by the nanoscopic model. For example, we vary the temperature from the average minimum to the average maximum temperature recorded in Europe with a step of 1 degree Celsius (Tank and Konnen, 2003).

We evaluate the energy consumption on a simplified route to minimize the computation time. The bus starts from a depot, stops at a bus stop and then returns to depot travelling $12 \mathrm{~km}$ in total. The bus accelerates uniformly until it reaches a specified top speed, and then it cruises at the same speed until it decelerates to the next stop with uniform deceleration. The created speed profile has a trapezoid shape. The model outputs the travelled distance and the accumulated energy consumption with a 0.1 second frequency.

It is crucial to separate the energy consumption into two categories, namely, traction and auxiliary. Traction consumption is linked to the movement of the vehicle, and it can be expressed in kilowatt hour $(\mathrm{kWh})$ per kilometer. Meanwhile, the auxiliary consumption is linked to energy used by the auxiliary system installed on the bus. Auxiliaries account for heating, ventilation, air conditioning, internal bus displays and lights among others. It is calculated per unit of time, and it is independent from the distance travelled. This can be expressed in kilowatt $(\mathrm{kW})$. 


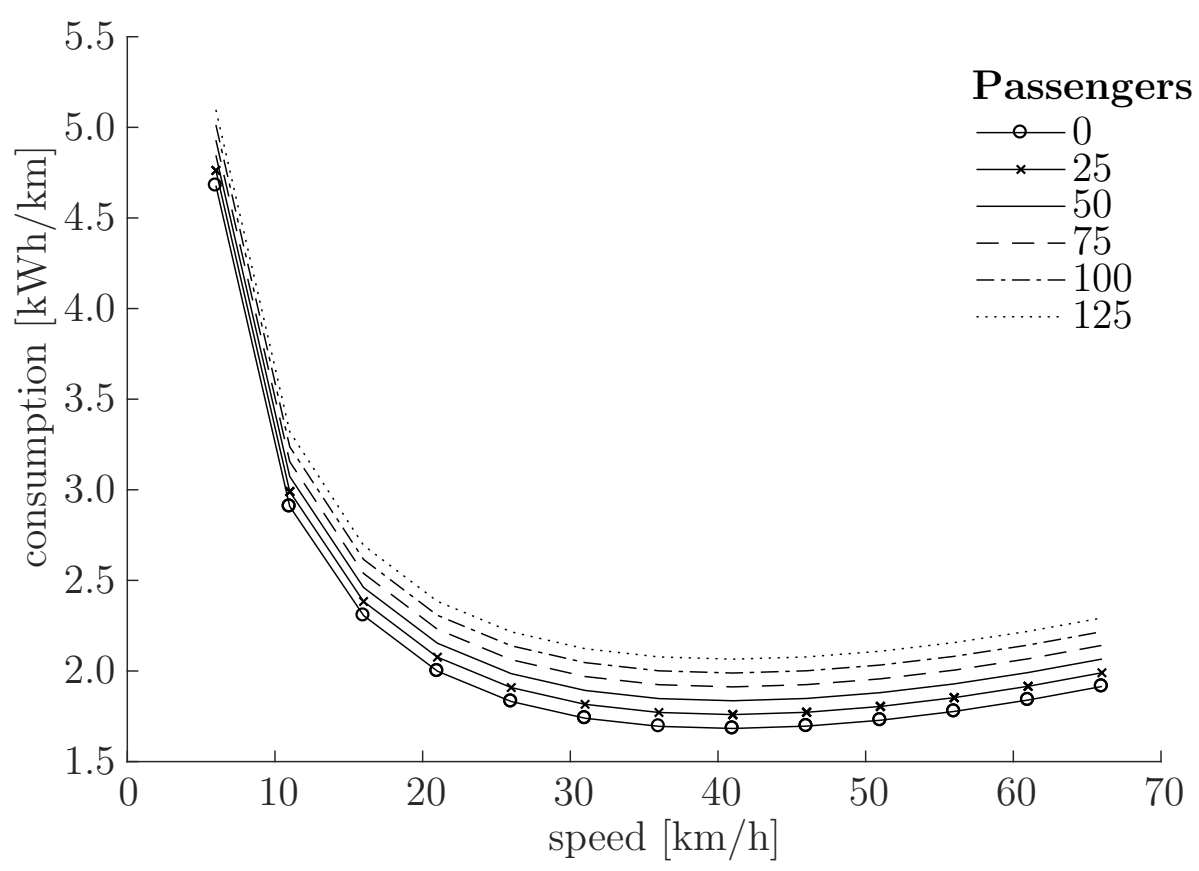

Figure 2: Example of energy consumption induced by the variations of two parameters: speed and number of passengers.

This distinction is important to estimate the vehicle consumption correctly. For example, when a vehicle is at a bus stop, its traction consumption expressed in $\mathrm{kWh}$ per kilometer travelled is undefined given that the speed is zero, but the bus still has an auxiliary consumption proportional to the stopping time.

Results and estimation. We obtain the energy consumption data varying the parameter values. Figure 2 shows an example of the traction energy consumption varying the bus speed and the number of passengers.

With the resulting data, we specify a polynomial regression model using least squares fitting with selected parameters as predictors, such as speed, acceleration, number of passengers on board, initial state of charge, street slope and temperature. The fitted model approximates the output of the nanoscopic energy consumption model. We separate the database into two parts, one is used for fitting the regression model (containing 65,000 points), while the other part is used for cross-validation (containing 29,000 points). The cross-validation set is used to examine the model overfitting. We analyze the model using the coefficient of determination, mean-squared-error and root-mean-square error as well as residual analysis.

We test different model specifications to identify the most appropriate order of polynomial regression. We generate a model for each polynomial degree from 1 to 5 and observe their coefficient of determination $\left(\mathrm{R}^{2}\right)$ and their root-meansquare-error (RMSE). As an example, Eq. (1) represents the fitted second order polynomial model composed of 15 coefficients. The variables $x_{1}-x_{4}$ are the first four components of the principal component analysis (PCA) performed on the 
Table 1: Comparison of polynomial regressions of different order

\begin{tabular}{c|c|c|c|c|c} 
& First & Second & Third & Fourth & Fifth \\
\hline Coefficient of determination & 0.8127 & 0.9315 & 0.9582 & 0.9693 & 0.9739 \\
RMSE & 1.9579 & 1.1811 & 0.9249 & 0.7924 & 0.7306
\end{tabular}

Table 2: Maximum absolute deviation and mean-squared-error for the generating and cross-validation data sets

\begin{tabular}{l|c|c|c} 
Generating data & Third & Fourth & Fifth \\
\hline Maximum absolute deviation & 4.6869 & 5.5819 & 3.8299 \\
Mean-squared-error & 0.8558 & 0.6285 & 0.5348 \\
& & & \\
Cross-validation data set & Third & Fourth & Fifth \\
\hline Maximum absolute deviation & 3.9101 & 4.7511 & 4.8364 \\
Mean-squared-error & 1.5969 & 1.6005 & 1.3003
\end{tabular}

generating dataset.

$$
\begin{aligned}
f\left(x_{1}, x_{2}, x_{3}, x_{4}\right)= & -9.1829 \mathrm{E}-06 x_{1}^{2}+8.8893 \mathrm{E}-03 x_{1} x_{2} \\
& +1.1358 \mathrm{E}-3 x_{1} x_{3}-1.5992 \mathrm{E}-4 x_{1} x_{4} \\
& +8.7243 \mathrm{E}-3 x_{1}-2.6577 x_{2}^{2}-0.27281 x_{2} x_{3} \\
& -4.9257 \mathrm{E}-3 x_{2} x_{4}+8.0492 x_{2}-4.1905 \mathrm{E}-3 x_{3}^{2} \\
& +2.5617 \mathrm{E}-2 x_{3} x_{4}+0.18687 x_{3}+6.9064 \mathrm{E}-3 x_{4}^{2} \\
& -0.43198 x_{4}+7.5115 .
\end{aligned}
$$

The results of $\mathrm{R}^{2}$ and RMSE for the different models are listed in Table 1 . We also report a more detailed analysis of the third, fourth and fifth order polynomial regressions. Table 2 shows the maximum absolute deviation and mean-squarederror from the generating set and the cross-validation set. From these results, we conclude that the fifth order polynomial model is the preferred choice. Although, it has a higher maximum deviation in cross-validation, it has a better fit in term of mean-squared-error for the generating and cross-validation sets. We plot the residuals for this model in Figure 3 for a graphical analysis of the residual distribution. The image confirms the good fit of the model for our purpose.

The estimated function is used by the traffic simulation to approximate the bus consumption over a large number of scenarios. The scenarios with the most critical KPI values can be re-evaluated with a direct call to the nanoscopic energy consumption to obtain more accurate results.

This approach brings a great flexibility in testing different dimensioning decisions. If the underlying nanoscopic model changes, this change can be reflected to the macroscopic model. For example, if a different model of car is used for an electric car-sharing system, or a different size of the battery pack of an electric 


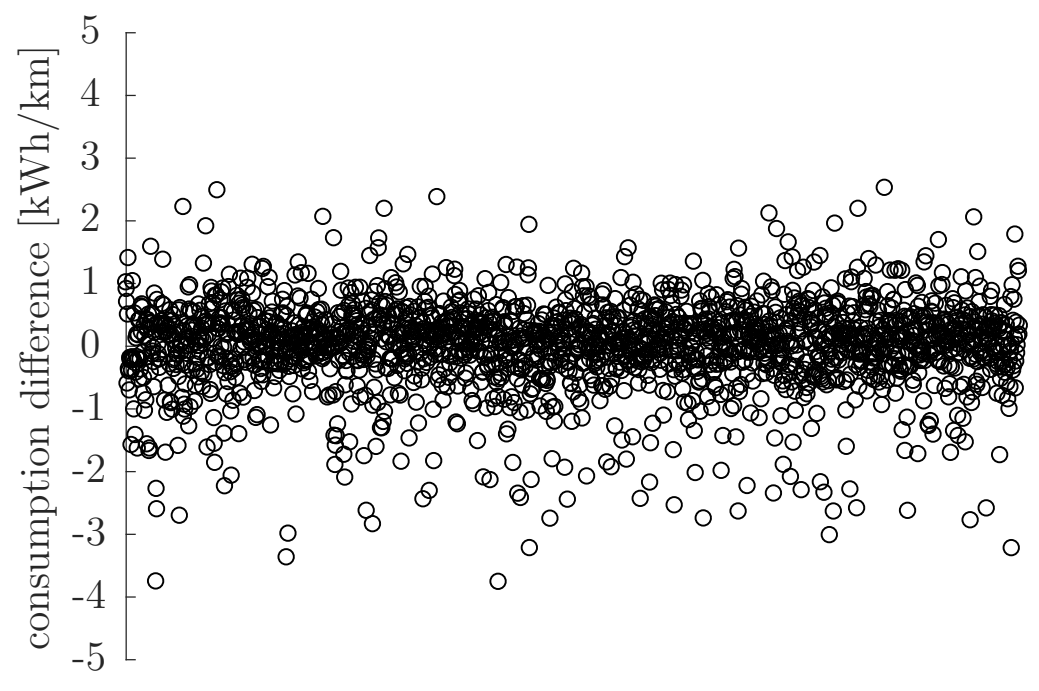

Figure 3: Residual plot of the order five model for the cross-validation set.

bus is chosen, this modification can be included in the nanoscopic model, and a new macroscopic model can be estimated. A good practice is to implement an automatic procedure for the estimation of the macroscopic model. In this way, a new model can be automatically estimated for different dimensioning decisions to be evaluated in the traffic simulation.

\section{Representation of the stopping profile}

Energy consumption is strongly related to the acceleration and deceleration of the vehicle rather than the average speed. The same average speed can be achieved with different speed profiles. For example, a trapezoid speed profile with only one acceleration and deceleration phase and a low cruising speed, or a complex speed profile with several intermediate stops and consequent acceleration/deceleration phases, and high top speeds, can have the same average speed.

Speed profiles are functions of many factors, such as intersections (including traffic lights, stop and priority signs, and roundabouts), slopes and congestion. The location of intersections is the main factor influencing the number of stops, and, as a consequence, the energy consumption (Fernandez, 2010). The number and location of intersections along the road network is unknown a priori for the early planning stage or for unknown routes. For example, a route chosen by a car-sharing customer is unknown, although the expected distance or travel time is given when the car is booked. Therefore, we need to represent intersections as random variables.

In this section, we define the probability distribution of the distance between two intersections. With this information, we can then generate the location of intersections in a road network given the probability distribution of the distance 


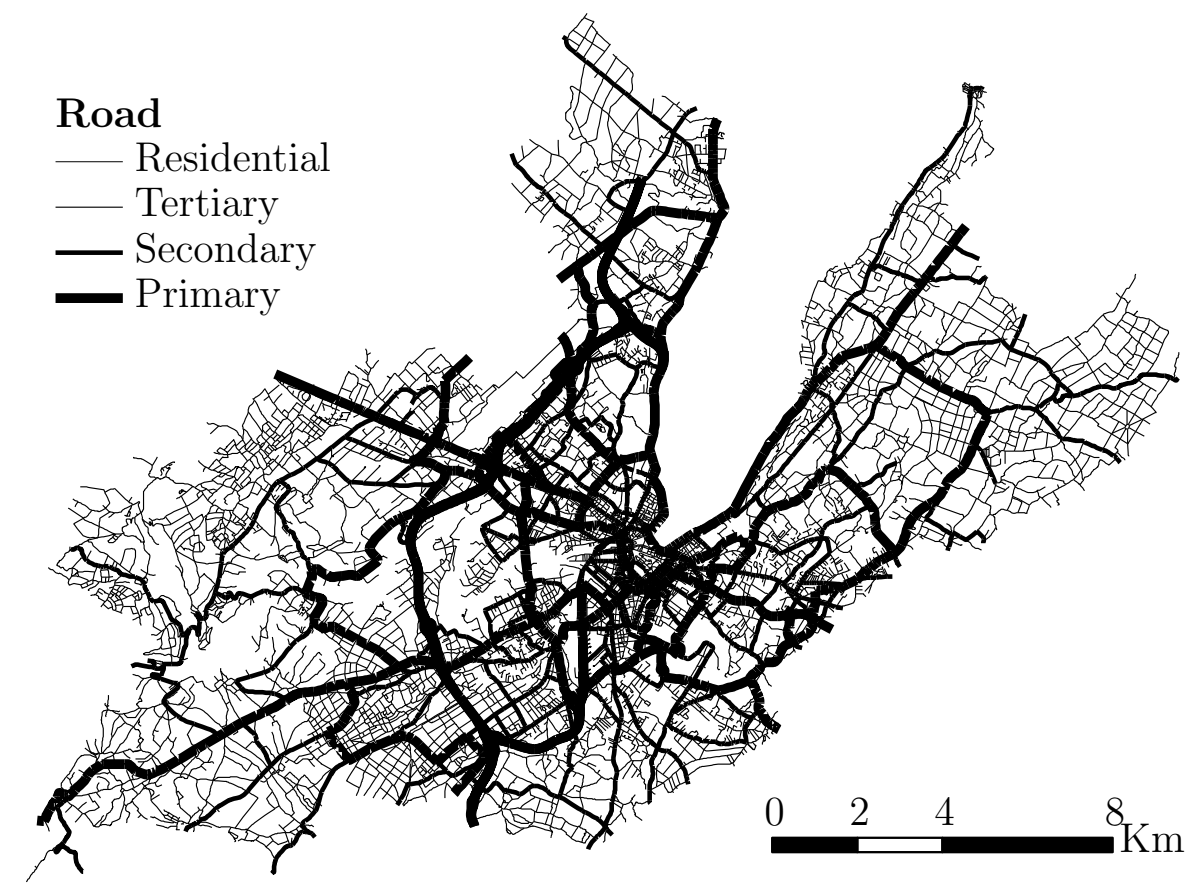

Figure 4: Network of Geneva with road classification used for the intersection distribution estimation.

between them.

We define an intersection as a location where a vehicle could potentially stop. Note, that this implies that the definition of intersection is both geometrical and hierarchical. In the case of stop and priority signs, when a primary road crosses a secondary road, vehicles traveling on the primary road have priority. This means that no "probability of stopping" is present for the vehicles travelling on the primary road. The intersection is present only on the secondary road. Following this consideration, we define an intersection along a road when the road crosses another road with a higher or equal hierarchy.

This definition may underestimate the number of possible stops. This is because a vehicle traveling on a main road crossing a small road may still have to stop. For example, it is possible that a traffic light or a roundabout is present at the intersection, which gives the possibility for the traffic from the small road to cross. However, we use the given definition of intersection because the information on the type of intersections is typically not available in the early planning stage.

We use the network of the city of Geneva, Switzerland, as a base study to define the probability distribution of the distance between intersections for different types of road, namely primary, secondary, tertiary and residential roads. We restrict our area of study to the urban area of the municipality because we focus on electric urban mobility. The road network is provided by the geographical information system of the canton of Geneva (SITG, 2015). Figure 4 represents the road network used with the road classification.

Following the previous definition of intersection, we separate the network based on the type of roads to build a hierarchy map. First, we select a network only 


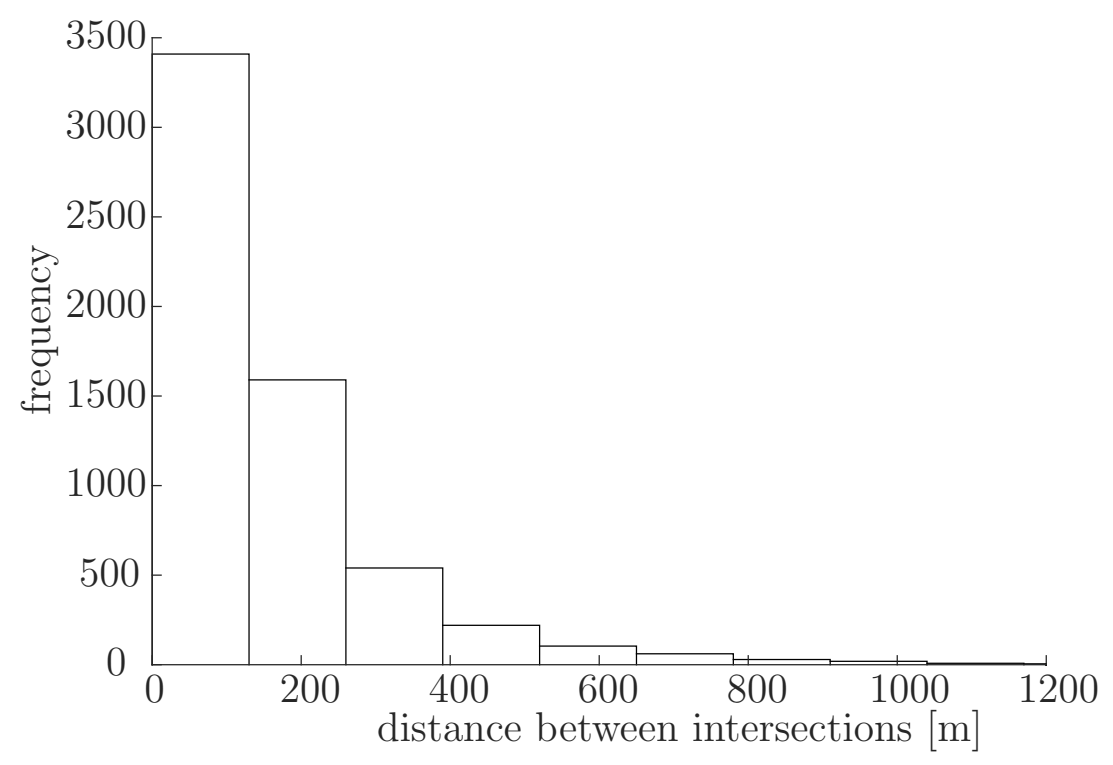

Figure 5: Histogram of the distances between intersections for all road types.

with primary roads. Then, we build a network with secondary and primary roads, and we use it to calculate the intersections on secondary road. Then, we build a network with tertiary, secondary and primary roads, and so on. We calculate the distances between intersections as the length of the segments of the created networks. We plot the distances obtained for all types of roads in Figure 5.

We now identify the probability distribution that better fits the data. The only requirement for the distribution is that it has a positive support, given that the distance between intersections cannot be negative. We test three different probability distributions: lognormal, gamma and exponential. We estimate the parameters of the distributions using maximum log likelihood, and we select the best distribution based on the Bayesian Information Criterion (BIC). The minimum BIC indicates the best fitting curve to our data, considering both goodness of fit and the number of parameters.

Table 3 reports the fitting results for different model specifications for all roads and individual road types. It is visible that the lognormal distribution has the lower BIC in all cases. The parameters $\mu$ and $\sigma$ for each lognormal distribution are reported in Table 3, and the distributions are plotted in Figure 6.

The results show an expected behavior. Residential roads are characterized by a short distance between intersections. The distance increases for tertiary and secondary roads, and primary roads have a large distance. We observe that the curve for primary roads strongly differs from the others. It is flatter and has a larger probability of great distance between intersections. It is also interesting to notice that the model for all types of roads is similar to the secondary and tertiary roads. This means that this model is representative of the intersection distribution in the road network, and it is not an average atypical case. The results are also consistent with the values found in the literature reviewed in Section 2.

A limitation of the case study is that the geographical database used does not 
Table 3: BIC and lognormal parameters for type of road and model specification

\begin{tabular}{lllll} 
& & \multicolumn{3}{c}{$\begin{array}{c}\text { Parameters } \\
\text { lognormal }\end{array}$} \\
Road type & Model & BIC & $\mu$ & $\sigma$ \\
\hline All types & Lognormal & 74514 & 4.7285 & 0.8538 \\
& Gamma & 74561 & & \\
& Exponential & 75377 & & \\
\hline Primary & Lognormal & 896.4 & 6.3332 & 1.1964 \\
& Gamma & 906.8 & & \\
& Exponential & 904.0 & & \\
\hline Secondary & Lognormal & 12834 & 4.8541 & 1.2562 \\
& Gamma & 13158 & & \\
& Exponential & 13243 & & \\
\hline Tertiary & Lognormal & 23217 & 4.7032 & 1.1034 \\
& Gamma & 23647 & & \\
& Exponential & 23649 & & \\
\hline Residential & Lognormal & 26694 & 4.3736 & 1.2755 \\
& Gamma & 27189 & & \\
& Exponential & 27326 & & \\
\hline
\end{tabular}

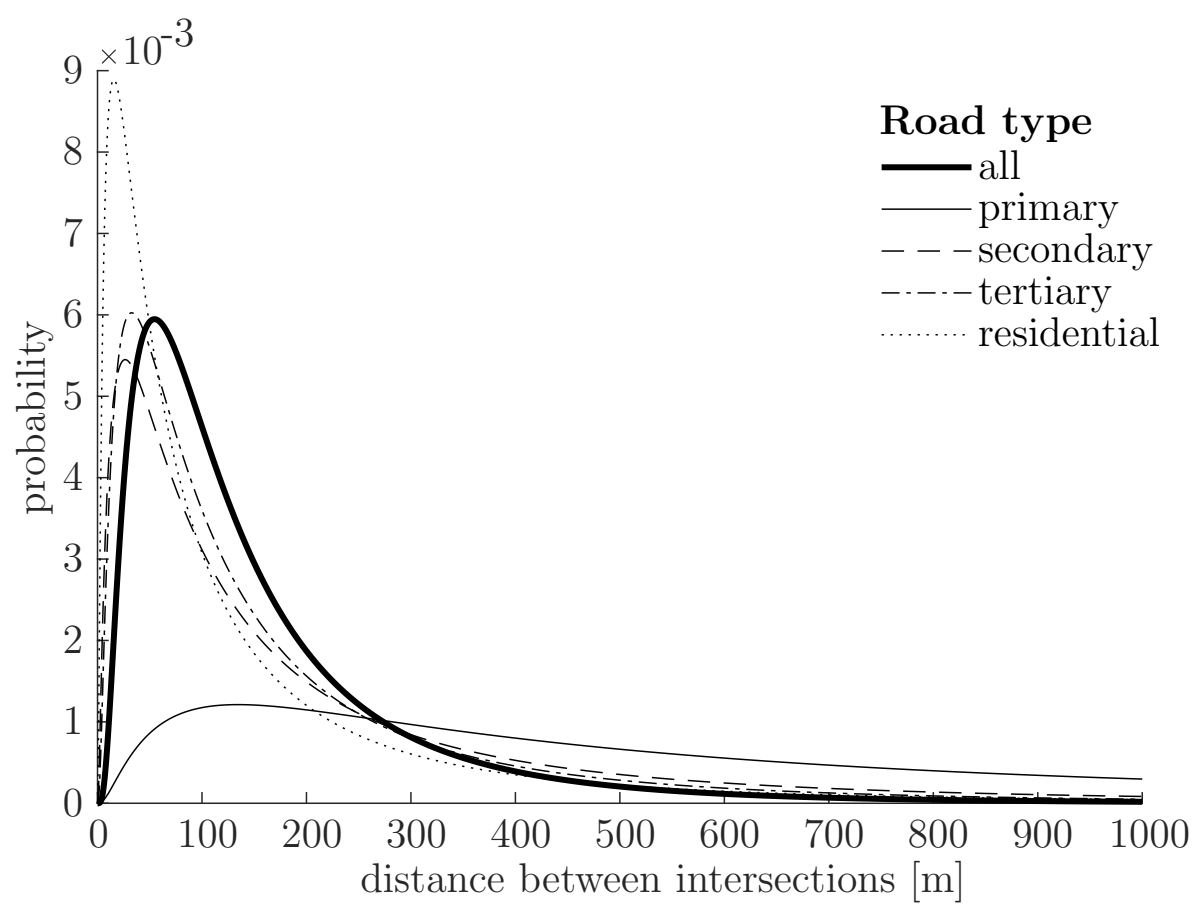

Figure 6: Fitted lognormal model for each road type. 
include information on the type of intersection crossed, e.g. traffic light, roundabouts or stops. Different types of intersections induce different speed profiles. This information can be accommodated in the presented methodology adding additional hierarchical layers.

The model representing the location of the intersections is integrated in the traffic simulation. A different set up of number and locations of intersections is generated at each run. The information on the intersection is combined with other realizations of the stochastic inputs (e.g. the traffic condition modifies the probability of stopping at a given intersection) to generate a specific speed profile of a simulation run.

This procedure is useful in the early planning stage to provide a rough feasibility of the dimensioning decisions.

\section{Application on a catenary-free electric bus ser- vice}

We integrate all presented aspects in the traffic simulation framework, and we show a possible use of this tool in a real case study: a bus line served by a catenary-free bus in Geneva.

We assume that a transport planner is interested in the minimum battery state of charge (SoC) over the entire vehicle schedule. This depends on all input characteristics, such as passengers, weather conditions, driver characteristics, traffic conditions, intersection locations and the resulting speed profile.

We simulate a bus line with 29 bus stations, 37 stands, 112 arcs, 2 terminal and 1 depot. We use 1,000 simulation runs, and we obtain the KPI for each run. Thanks to the structure of the simulator, the total computation time is less than 4 seconds.

We report the results in Figure 7. The image shows the empirical distribution of the minimum SoC of the on-board battery for all simulation runs.

The generated distribution of the SoC is crucial for taking informed dimensioning decisions. For example, let us assume that the dimension decision is the battery size, and the minimum acceptable state of charge of the battery is $35 \%$ at any time. The results of the traffic simulation quantify how often the minimum $\mathrm{SoC}$ is below this threshold. In our case, it happens $4.4 \%$ of the times. This proportion is represented by the dark area in Figure 7. This information considers extreme cases and facilitates the risk assessment of the dimension decisions.

We now show how the simulation can be used to evaluate different strategies to reduce the probability of low SoC. Let us assume that the transport planner considers the current probability that the minimum on-board battery SoC is below the threshold too high. As mentioned, the probability that this happens is $4.4 \%$ with the current system configuration.

The transport planner evaluates two strategies: strategy-1 "driver education", strategy-2 "battery improvement". Strategy-1 aims at improving the drivers' driving style. In particular, this strategy educates drivers in applying a similar ac- 


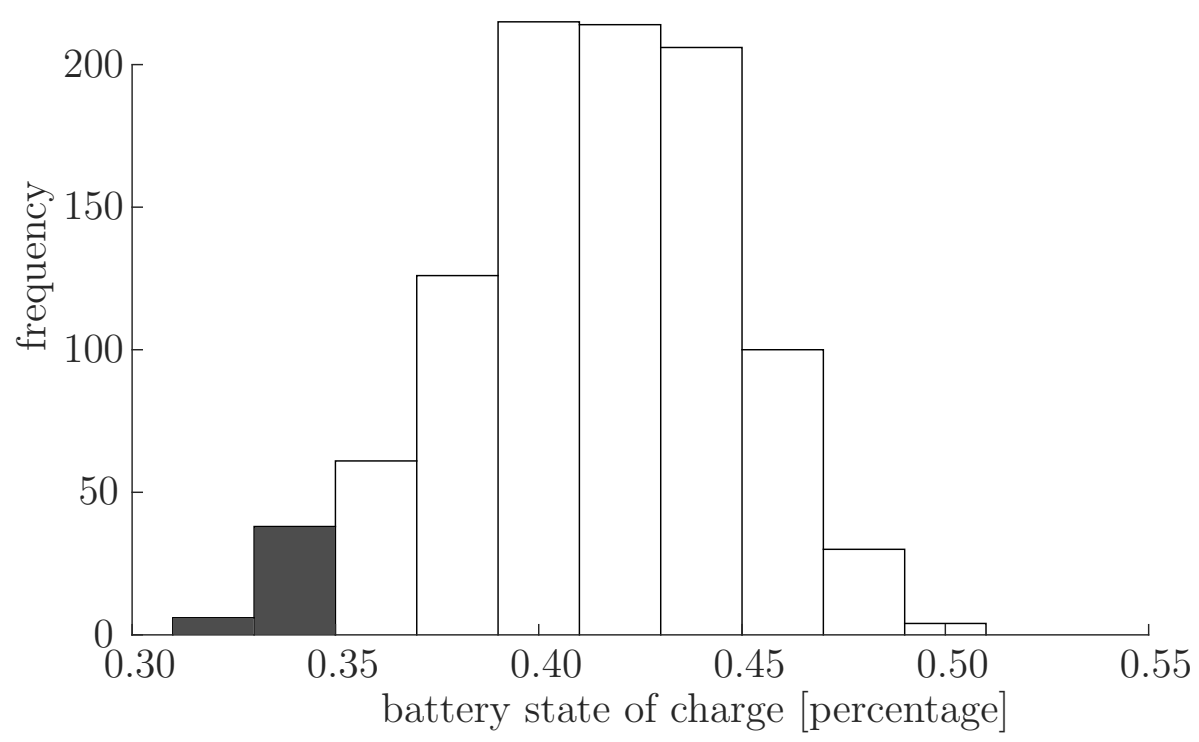

Figure 7: Minima battery state of charge histogram for 1,000 runs, and representation of the area below an acceptable threshold of 35\% state of charge.

celeration every time. This leads to a lower variability in the bus acceleration, which is coded in the simulation software as a reduction of $10 \%$ of the standard deviation of the driver desired acceleration. Strategy-2 is a dimensioning decision, which consist of increasing the capacity of the on-board battery of $1 \%$.

We evaluate the consequence on the KPI by running the simulation with two different configurations, one for each strategy. The results for 1,000 runs indicates that strategy-1 leads to SoC below the threshold of $2.0 \%$, and strategy-2 leads to SoC below the threshold of $2.6 \%$. Moreover, the empirical distribution of the minimum SoC has a lower variance, as shown in Figure 8 and Figure 9 in comparison to Figure 7 . Both strategies successfully reduce the probability of low SoC, and the decision of which one is the more effective includes costs, feasibility and strategical considerations to be evaluated by the traffic planner.

Besides the results of the KPIs, the simulation provides useful information on the operations of the buses. For example, it is possible to analyze the arrival times at the bus stops. Each bus tries to follow a time schedule, however, due to traffic, passenger embarking/disembarking and recharging operations, the bus could encounter delays. The simulation record the "effective" arrival time at each stop, and we can compare with the scheduled one. Figure 10 shows the histogram of these differences for 1,000 simulation runs. We can see that the majority of the times, the buses arrive within a few seconds from the scheduled time. This is the central bin around the value of zero time difference. Delays larger than 60 seconds happen rarely, only on $5.6 \%$. On the contrary, it is more probable that the bus arrives at the stop before schedule. The probability that the bus arrives more than 60 seconds in advance is $19.7 \%$. This indicates that the bus schedule can be respected the vast majority of the times.

It is clear that the proposed framework offers more information to the decision maker in comparison with the methods used as current state of practice, which 


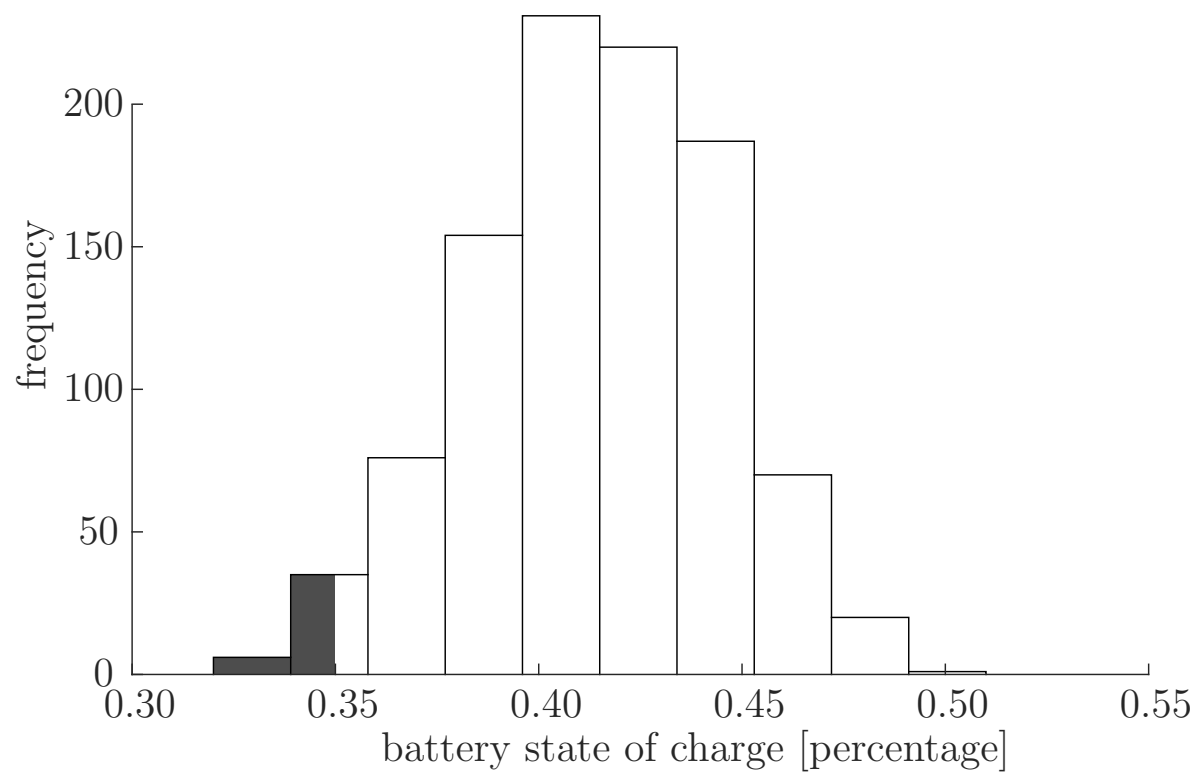

Figure 8: Minima battery state of charge histogram for strategy-1 "driver education".

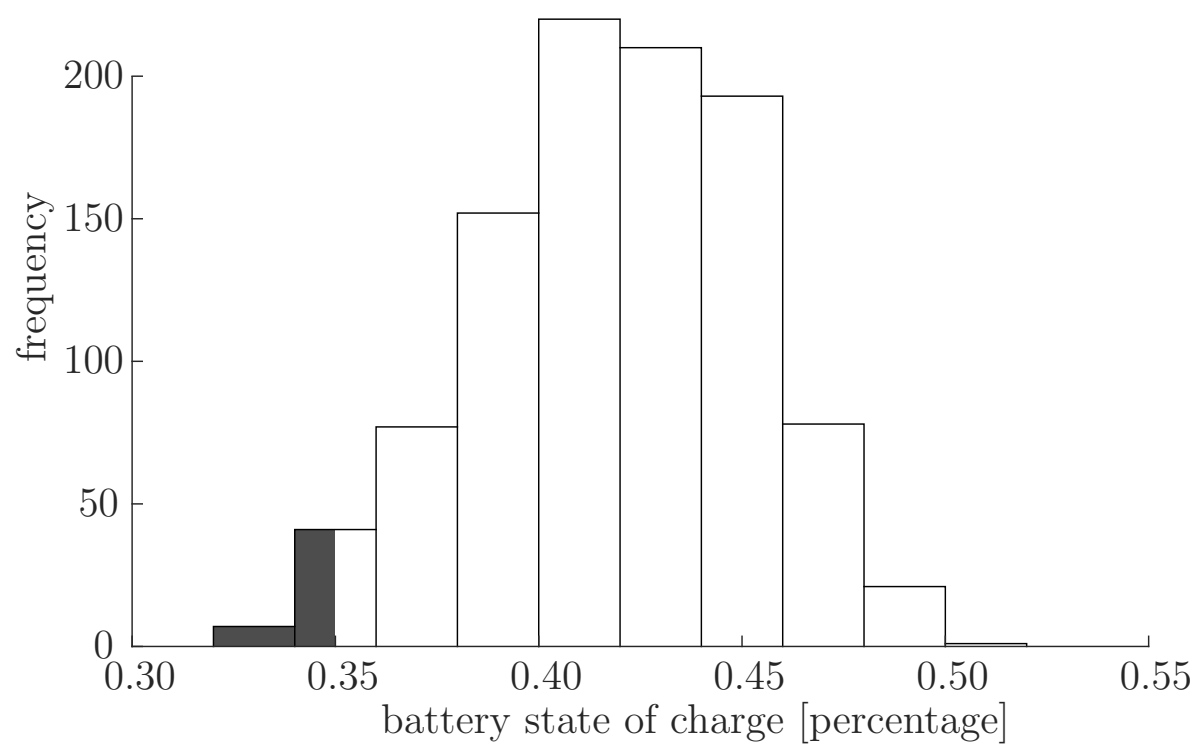

Figure 9: Minima battery state of charge histogram for strategy-2 "battery improvement". 


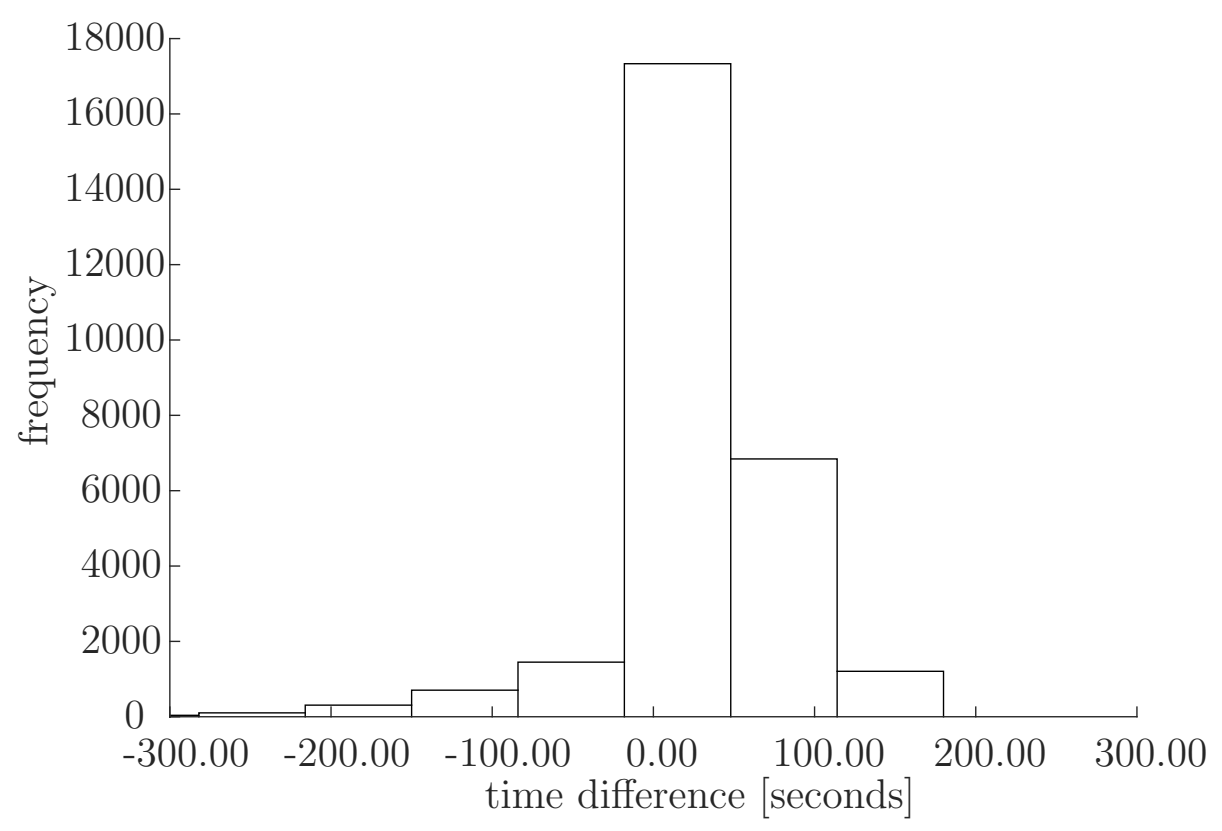

Figure 10: Time difference between scheduled and executed arrival time. Negative values indicate a delay in the arrival, and positive values indicate an arrival before the scheduled time.

provide only a single value of the KPI indicating the performance of the system based on the average case.

We use this application to show the potential of the framework. However, a rigorous calibration and validation of the simulation should be done using empirical data, possibly, from different case studies and vehicle types. For the presented case study, we perform the calibration of the traffic simulation on macroscopic characteristics. Examples of available information at the early planning stage is the average commercial speed and the expected delay distribution of vehicle at bus stops shown in Figure 10.

\section{Conclusions}

We develop a traffic simulation framework useful to simulate the operations of electric vehicles. We describe the structure, the main components and the logical interactions among them. Elements are represented at different modeling scales and, when there is a lack of information, they are represented as random variables, like in the case of the stopping profile. This approach is robust to missing data and allows evaluating the key performance indicators in an early stage of the planning phase. Thanks to the integration of a macroscopic representation of the nanoscopic energy consumption model, we can evaluate a multitude of scenarios in a short computation time. This allows the identification of extreme cases that require a more accurate evaluation. The simulation returns the distribution of the KPIs, which is a valuable information for transport planners, as shown in the case of a catenary-free bus service. 
The framework developed here can be useful for the simulation of electric vehicles in general. The need for this type of tools is particularly evident given the forecast of a large deployment of electric vehicles, both private and public, in the next years. For example, the framework can be used for simulating the management of an electric car-sharing system in combination with the feeding station locations, or the management of a fleet of autonomous electric buses.

The main challenge of electric mobility is the recharging of the on-board battery. This operation is critical because it needs a long period of time, and a miscalculation of the battery state of charge can lead to severe consequences on the operation of the system. This greater fragility, not present in services using traditional vehicles, which refuel in a matter of minutes, calls for more precise planning tools. For this reason, planning tools based on simulation, like presented in this paper, are more appropriate given their capability of evaluating extreme cases and giving probability distribution of the key performance indicators, and they can facilitate the transition toward the electrification of urban mobility.

The main direction for future research is the integration of the traffic simulation in the methods used for taking dimensioning decisions. For example, simulationbased optimization can be used instead of optimization models such as mixedinteger linear programming (MILP) that rely on the average performance of the system. This approach, considering the stochasticity of the inputs and evaluating extreme cases, could improve the planning of transport systems.

\section{Acknowledgment}

This research is part of the project "myTOSA 2.0 - Enhanced simulation and optimization tool for cost-optimal deployment of TOSA electric buses (18138.1 PFES-ES)" funded by the Swiss Commission for Technology and Innovation CTI. We thank Romain Meyer, Xiaoran Yu, Adrien Nicolet and Axel Valentin for their contributions on this research.

\section{References}

Antrim, A., Barbeau, S.J., 2013. The many uses of GTFS dataopening the door to transit and multimodal applications. Location-Aware Information Systems Laboratory at the University of South Florida, 4.

Banister, D., 2001. Transport planning. Handbook of transport systems and traffic control, Emerald Group Publishing Limited.

Barcelo, J., 2010. Fundamentals of traffic simulation. volume 145. Springer.

Bierlaire, M., 2015. Simulation and optimization: a short review. Transportation Research Part C: Emerging Technologies 55, 4-13.

Black, J., 2018. Urban transport planning: Theory and practice. Routledge. 
Cadarso, L., Codina, E., Escudero, L.F., Marín, A., 2017. Rapid transit network design: considering recovery robustness and risk aversion measures. Transportation Research Procedia 22, 255-264.

Cardillo, A., Scellato, S., Latora, V., Porta, S., 2006. Structural properties of planar graphs of urban street patterns. Physical Review E 73, 066107.

Chen, J., Bierlaire, M., Robenek, T., 2014. myTOSA: simulation tool for the dimensioning, commercial promotion and case study set-up for ABB's revolutionary catenary-free $100 \%$ electric urban public mass-transportation system. Technical Report TRANSP-OR 140430. EPFL.

Cimini, G., Kim, Y., McCain, B., Siegel, J., Stefanopoulou, A., 2017. Model Predictive Control for Real-time Position Tracking of a Catenary-free Tram. IFAC-PapersOnLine 50, 1000-1005.

Crucitti, P., Latora, V., Porta, S., 2006. Centrality measures in spatial networks of urban streets. Physical Review E 73, 036125.

Deb, K., Chakroborty, P., 1998. Time scheduling of transit systems with transfer considerations using genetic algorithms. Evolutionary computation 6, 1-24.

Desaulniers, G., Hickman, M.D., 2007. Public transit. Handbooks in operations research and management science 14, 69-127.

Dunn, J., Gaines, L., Kelly, J., James, C., Gallagher, K., 2015. The significance of Li-ion batteries in electric vehicle life-cycle energy and emissions and recycling's role in its reduction. Energy and Environmental Science 8, 158-168.

Ehsani, M., Gao, Y., Longo, S., Ebrahimi, K., 2018. Modern electric, hybrid electric, and fuel cell vehicles. CRC press.

Erkkila, K., Nylund, N.O., Pellikka, A.P., Kallio, M., Kallonen, S., Ojamo, S., Ruotsalainen, S., Pietikinen, O., Lajunen, A., 2013. eBUS-Electric bus test platform in Finland, in: Proceedings of the 27th international electric vehicle symposium and exhibition (EVS27), Barcelona, Spain.

Fan, W., Machemehl, R.B., 2006. Optimal transit route network design problem with variable transit demand: genetic algorithm approach. Journal of Transportation Engineering 132.

Farahani, R.Z., Miandoabchi, E., Szeto, W.Y., Rashidi, H., 2013. A review of urban transportation network design problems. European Journal of Operational Research 229, 281-302.

Fernandez, R., 2010. Modelling public transport stops by microscopic simulation. Transportation Research Part C: Emerging Technologies 18, 856-868.

Fernandez-Antolin, A., de Lapparent, M., Bierlaire, M., 2016. Modeling purchases of new cars: an analysis of the 2014 French market. Theory and Decision , 1-27. 
Filippo, G.D., Marano, V., Sioshansi, R., 2014. Simulation of an electric transportation system at The Ohio State University. Applied Energy 113, 1686-1691.

Frade, I., Ribeiro, A., Goncalves, G., Antunes, A., 2011. Optimal location of charging stations for electric vehicles in a neighborhood in Lisbon, Portugal. Transportation research record: journal of the transportation research board, 91-98.

Gao, D.W., Mi, C., Emadi, A., 2007. Modeling and simulation of electric and hybrid vehicles. Proceedings of the IEEE 95, 729-745.

Guihaire, V., Hao, J.K., 2008. Transit network design and scheduling: A global review. Transportation Research Part A: Policy and Practice 42, 1251-1273.

He, F., Wu, D., Yin, Y., Guan, Y., 2013. Optimal deployment of public charging stations for plug-in hybrid electric vehicles. Transportation Research Part B: Methodological 47, 87-101.

Hess, A., Malandrino, F., Reinhardt, M.B., Casetti, C., Hummel, K.A., BarcelOrdinas, J.M., 2012. Optimal deployment of charging stations for electric vehicular networks, in: Proceedings of the first workshop on Urban networking, pp. 1-6.

Horsky, M., 2018. Bus simulation module for enhanced simulation and optimization tool for cost-optimal deployment of TOSA electric buses. Technical Report. Computational Mechanics group, Haute-Ecole ARC Ingnierie.

Hu, X., Johannesson, L., Murgovski, N., Egardt, B., 2015. Longevity-conscious dimensioning and power management of the hybrid energy storage system in a fuel cell hybrid electric bus. Applied Energy 137, 913-924.

Jacobs, A.B., 1993. Great streets. ACCESS Magazine 1.

Jansen, L.N., Pedersen, M.B., Nielsen, O.A., 2002. Minimizing passenger transfer times in public transport timetables, in: 7th Conference of the Hong Kong Society for Transportation Studies, pp. 229-239.

Jiang, B., Claramunt, C., 2004. Topological analysis of urban street networks. Environment and Planning B: Planning and design 31, 151-162.

Kuhne, R., 2010. Electric busesAn energy efficient urban transportation means. Energy 35, 4510-4513.

Larminie, J., Lowry, J., 2004. Electric vehicle technology explained. John Wiley and Sons.

Lurkin, V., Zanarini, A., Maknoon, Y., Azadeh, S.S., Bierlaire, M., 2018. Planning of feeding station installment for a full electric large capacity urban bus system. Technical Report TRANP-OR 180301. EPFL. 
Marshall, S., 2004. Streets and patterns. Routledge.

Moreno, J., Ortzar, M.E., Dixon, J.W., 2006. Energy-management system for a hybrid electric vehicle, using ultracapacitors and neural networks. IEEE Transactions on Industrial Electronics 53, 614-623.

Nikolic, M., Teodorovic, D., 2013. Transit network design by bee colony optimization. Expert Systems with Applications 40, 5945-5955.

Palomares, V., Serras, P., Villaluenga, I., Hueso, K.B., Carretero-Gonzalez, J., Rojo, T., 2012. Na-ion batteries, recent advances and present challenges to become low cost energy storage systems. Energy and Environmental Science 5, $5884-5901$.

Peponis, J., Allen, D., Haynie, D., Scoppa, M., Zhang, Z., 2007. Measuring the configuration of street networks, in: 6th international space syntax symposium, pp. 1-16.

Perrotta, D., Macedo, J.L., Rossetti, R.J., de Sousa, J.F., Kokkinogenis, Z., Ribeiro, B., Afonso, J.L., 2014. Route planning for electric buses: a case study in Oporto. Procedia-Social and Behavioral Sciences 111, 1004-1014.

Punpaisan, S., Kulworawanichpong, T., 2014. Dynamic Simulation of Electric Bus Vehicle. Standard International Journals (SIJ) 2, 99-104.

Rahman, I., Vasant, P.M., Singh, B.S.M., Abdullah-Al-Wadud, M., Adnan, N., 2016. Review of recent trends in optimization techniques for plug-in hybrid, and electric vehicle charging infrastructures. Renewable and Sustainable Energy Reviews 58, 1039-1047.

Ross, S.M., 2013. Simulation. Academic Press.

Shirk, M., Wishart, J., 2015. Effects of electric vehicle fast charging on battery life and vehicle performance.

Silvas, E., Hofman, T., Murgovski, N., Etman, L.P., Steinbuch, M., 2017. Review of optimization strategies for system-level design in hybrid electric vehicles. IEEE Transactions on Vehicular Technology 66, 57-70.

SITG, 2015. Systeme d'information du territoire a Geneve. URL: http://ge.ch/ sitg/.

Tang, T.Q., Chen, L., Yang, S.C., Shang, H.Y., 2015. An extended car-following model with consideration of the electric vehicles driving range. Physica A: Statistical Mechanics and its Applications 430, 148-155.

Tank, A.K., Konnen, G., 2003. Trends in indices of daily temperature and precipitation extremes in Europe, 194699. Journal of Climate 16, 3665-3680. 
Walpole, S.C., Prieto-Merino, D., Edwards, P., Cleland, J., Stevens, G., Roberts, I., 2012. The weight of nations: an estimation of adult human biomass. $B M C$ public health 12, 439 .

Yang, S., Deng, C., Tang, T., Qian, Y., 2013. Electric vehicles energy consumption of car-following models. Nonlinear Dynamics 71, 323-329. 